\title{
Two Cases of Kikuchi Disease Presenting with Aseptic Meningitis and Encephalitis
}

\author{
Nobuki Iwamoto ${ }^{1}$, Mari Funahashi ${ }^{2}$, Koh Shinohara ${ }^{3}$, Yoshifumi Nakaya ${ }^{2}$, \\ Hirofumi Motobayashi ${ }^{1}$, Kentaro Tochitani ${ }^{1}$, Shungo Yamamoto ${ }^{1}$ and Tsunehiro Shimizu ${ }^{1}$
}

\begin{abstract}
:
Kikuchi disease, also called histiocytic necrotizing lymphadenitis, is an idiopathic and generally selflimiting disease affecting young adults and children. Kikuchi disease does not commonly manifest neurological complications at its initial presentation. We herein report two cases of Kikuchi disease that initially presented with aseptic meningitis and encephalitis rather than the more common signs of lymphadenopathy, rash, and arthritis. A 15-year-old boy presented with aseptic meningitis with an extremely high intracranial pressure. A 28-year-old man presented with dysesthesia of the right lower extremity, coinciding with abnormal magnetic resonance imaging findings. In both cases, painful cervical lymphadenopathy was observed following the central nervous system symptoms. Both patients improved after treatment with steroids. Kikuchi disease occasionally affects the central nervous system, to which lymphadenitis may be observed subsequently. A repeated, careful physical examination of the cervical lymph nodes may be helpful for the diagnosis.
\end{abstract}

Key words: Kikuchi disease, Aseptic meningitis, Encephalitis, Painful cervical lymphadenopathy, Neurological symptoms

(Intern Med Advance Publication)

(DOI: 10.2169/internalmedicine.7724-21)

\section{Introduction}

Kikuchi disease, also called histiocytic necrotizing lymphadenitis, is an idiopathic and generally self-limiting disease affecting young adults and children. The most common signs and symptoms include lymphadenopathy (100\%), a fever $(35 \%)$, erythematous rash $(10 \%)$, joint pain $(7 \%)$, fatigue (7\%), arthritis (5\%), and hepatosplenomegaly (3\%) (1). Neurological complications, including aseptic meningitis and encephalitis, are rare (1-3). Neurological complications manifesting at the initial presentation of Kikuchi disease are not well reported.

We herein report two cases of Kikuchi disease that initially presented with aseptic meningitis and encephalitis rather than the more common signs of lymphadenopathy, rash, and arthritis.

\section{Case Report}

\section{Case 1}

A 15-year-old Japanese boy with no significant medical history presented to a hospital with severe headaches and a remittent fever lasting approximately 2 months. During this time, the symptoms did not improve despite the oral administration of nonsteroidal anti-inflammatory drugs as needed. Lymphocytic pleocytosis $(70 / \mu \mathrm{L})$ was noted on a cerebrospinal fluid (CSF) examination. Head computed tomography (CT) and brain magnetic resonance imaging (MRI) findings were normal.

He was diagnosed with aseptic meningitis and underwent conservative treatment with acetaminophen for approximately one month. However, the patient's condition did not improve, so he was referred to our hospital for a further ex-

${ }^{1}$ Department of Infectious Diseases, Kyoto City Hospital, Japan, ${ }^{2}$ Department of Neurology, Kyoto City Hospital, Japan and ${ }^{3}$ Department of Clinical Laboratory Medicine, Kyoto University Graduate School of Medicine, Japan 

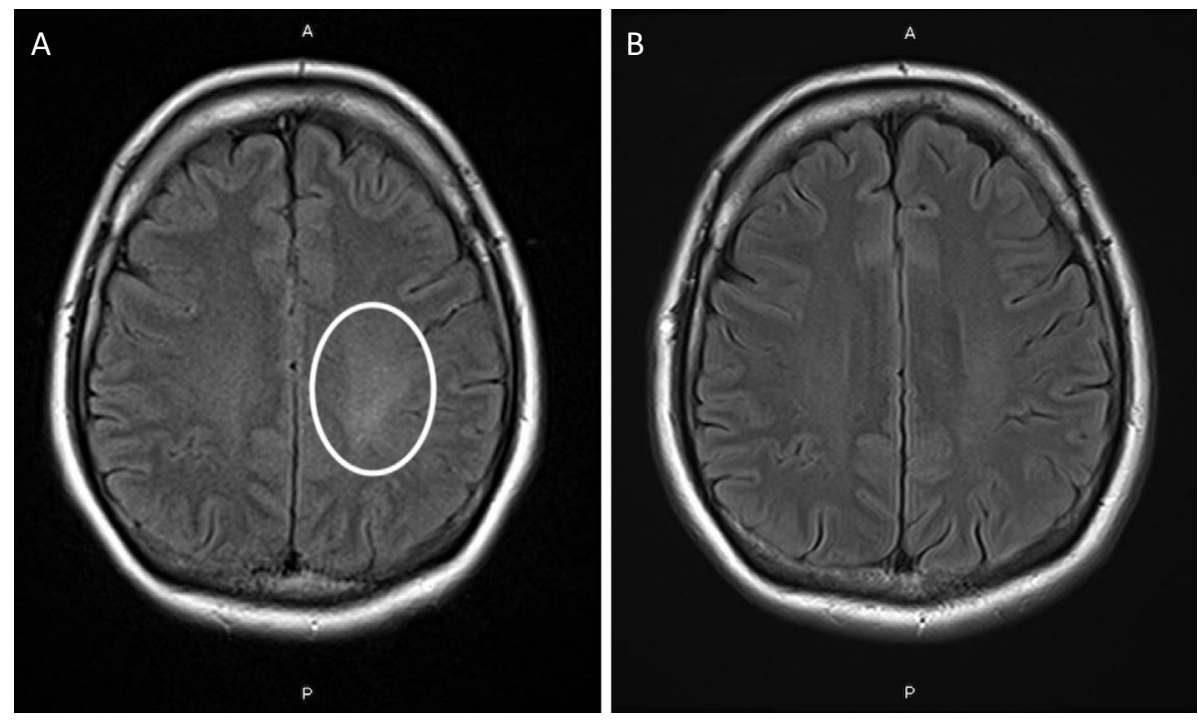

Figure. Transverse fluid-attenuated inversion recovery (FLAIR) -weighted imaging shows an area with a high signal intensity (circle) in the left parietal lobe (A). The high signal intensity disappeared after the administration of a steroid $(B)$.

amination and treatment. On admission, the patient was observed to have painful cervical lymphadenopathy that had persisted for a week. The CSF opening pressure was extremely high (over $30 \mathrm{mmH}_{2} \mathrm{O}$ ) but with normal findings for the cell count and glucose and protein levels. The CSF cultures for bacteria, fungi, and mycobacteria were negative. Histopathology of a cervical lymph node showed a sphenoid necrotic area with abundant nuclear debris and histiocytes, which are typical findings of histiocytic necrotizing lymphadenitis or Kikuchi disease. Based on the pathological and CSF findings, a diagnosis of aseptic meningitis associated with Kikuchi disease was made.

The patient was started on $30 \mathrm{mg}$ of oral prednisolone every day, which resulted in a significant improvement of his headache, fever, and cervical lymphadenopathy. The CSF opening pressure also decreased. The patient was discharged with a tapered dose of prednisolone. The steroid was further tapered and eventually discontinued one month later after symptoms of Kikuchi disease were no longer suspected. We followed the patient for approximately one month after discontinuing prednisolone, and within that time, there was no recurrence of Kikuchi disease.

\section{Case 2}

A 28-year-old Japanese man with no significant medical history presented to a hospital with a fever and dysesthesia of the right lower extremity that had lasted for approximately 2 months and 2 weeks, respectively. Brain MRI revealed white matter lesions in the left parietal lobe (Fig. A). He was referred to our hospital for a further examination and treatment. On admission, he was alert and had no focal neurological deficits other than dysesthesia of the right lower extremity. In addition, painful cervical lymphadenopathy that had persisted for two weeks was observed.

A physical examination did not reveal any findings of skin rash, oral ulcer, alopecia, arthritis, or Raynaud's phenomenon. Blood tests showed mild leukopenia $(3,460 / \mu \mathrm{L})$, anemia (11.9 g/dL), a normal platelet count $(185,000 / \mu \mathrm{L})$, and normal creatinine levels. The antinuclear antibodies were negative. A urinalysis revealed no abnormalities. In the CSF, lymphocytic pleocytosis $(69 / \mu \mathrm{L})$ was noted, with normal glucose and protein levels. The CSF cultures for bacteria, fungi, and mycobacteria were negative. Histopathology of a cervical lymph node showed a geographical necrotic area with abundant nuclear debris and histiocytes, which are typical findings of histiocytic necrotizing lymphadenitis. Although Kikuchi disease and systemic lupus erythematosus were considered in the differential diagnosis, we arrived at a diagnosis of Kikuchi disease because antinuclear antibodies were negative, and the histopathology was compatible with Kikuchi disease. Encephalitis associated with Kikuchi disease was suspected due to the dysesthesia of the right lower extremity, which was the focal symptom that corresponded with the abnormal MRI findings.

The patient was administered intravenous methylprednisolone at $1 \mathrm{~g}$ /day for 3 days. Follow-up brain MRI demonstrated the significant resolution of the white matter lesions that had been previously noted (Fig. B). The patient continued to improve clinically along with his CSF analysis findings. We had follow-ups with him for approximately eight months, during which there was no recurrence of encephalitis.

\section{Discussion}

We identified three important clinical issues in this study.

First, the initial presentation of Kikuchi disease can involve neurological complications, such as aseptic meningitis or encephalitis, occasionally preceding painful cervical lymphadenopathy. The development of meningitis in Kikuchi 
disease has been reported, but only a few cases report have described meningitis preceding other manifestations of Kikuchi disease (2). We herein report two cases of Kikuchi disease: one with an initial presentation of aseptic meningitis and the other with encephalitis. Cervical lymphadenopathy might have been present but was not observed when meningitis and encephalitis were suspected at the previous hospitals. Neither patient complained of painful or swollen lymph nodes prior to their neurological symptoms. In a previous report examining the time of the meningitis onset within the course of Kikuchi disease, 2 of 11 Kikuchi disease cases were preceded with meningitis by 2 and 3 weeks (2). In case 1 , the period between the onset of the neurological symptoms and that of painful lymphadenopathy was approximately three months, which was longer than that in the previous report.

Second, meningitis associated with Kikuchi disease may be accompanied by an extremely high intracranial pressure despite a normal CSF analysis. A case of Kikuchi disease with features of increased intracranial pressure, subsequently revealing bilateral subdural effusions, has been reported ${ }^{4}$. In this report, the dural biopsy showed histiocytic necrotizing inflammation identical to Kikuchi disease, confirming dural involvement (4). In case 1, the extremely high intracranial pressure without abnormal CSF analysis findings might have been caused by the slight involvement of the dura matter, although there were no intracranial lesions on head CT.

Third, we encountered an unusual case of encephalitis associated with Kikuchi disease. There are few cases of encephalitis accompanied by Kikuchi disease (5-9). Byun et al. reported the clinical and MRI findings of nine patients with encephalitis associated with Kikuchi disease (9). In that report, MRI abnormalities were often found in the cerebellum, brainstem, temporal lobes, and occipital lobes. Neurological symptoms included headaches, seizures, drowsiness, and an altered mental status. The duration between the onset of lymphadenopathy and central nervous system symptoms ranged from 10 to 53 days. In case 2, the patient experienced dysesthesia of the right lower extremity, which was the focal symptom corresponding to an abnormality of the left parietal lobe. Dysesthesia and lesions of the left parietal lobe in encephalitis associated with Kikuchi disease were not described in the report by Byun et al.
In conclusion, the initial presentation of Kikuchi disease can involve neurological complications, such as aseptic meningitis and encephalitis. Meningitis associated with Kikuchi disease may be accompanied by an extremely high intracranial pressure without abnormal CSF or intracranial lesions. We encountered a case with unusual symptoms and MRI abnormalities with encephalitis associated with Kikuchi disease. Kikuchi disease occasionally affects the central nervous system, and cervical lymphadenopathy may appear subsequently, even if not initially observed. A repeated, careful physical examination of the cervical lymph nodes may be helpful for the diagnosis.

The authors state that they have no Conflict of Interest (COI).

\section{References}

1. Kucukardali Y, Solmazgul E, Kunter E, Oncul O, Yildirim S, Kaplan M. Kikuchi-Fujimoto Disease: analysis of 244 cases. Clin Rheumatol 26: 50-54, 2007.

2. Sato Y, Kuno H, Oizumi K. Histiocytic necrotizing lymphadenitis (Kikuchi's disease) with aseptic meningitis. J Neurol Sci 163: 187191, 1999.

3. Khishfe BF, Krass LM, Nordquist EK. Kikuchi disease presenting with aseptic meningitis. Am J Emerg Med 32: 298, 2014.

4. Santos MV, Gallo P, Roked F, Nicklaus-Wollenteit I, Rodrigues D. Unusual presentation of Kikuchi-Fujimoto disease. J Neurosurg Pediatr 12: 266-269, 2013.

5. Kido H, Kano O, Hamai A, et al. Kikuchi-Fujimoto disease (histiocytic necrotizing lymphadenitis) with atypical encephalitis and painful testitis: a case report. BMC Neurol 17: 22, 2017.

6. Jasti DB, Naveen Prasad SV, Naveen T, Vengamma B. KikuchiFujimoto disease presenting as brain encephalitis with secondary blepharospasm. J Neurosci Rural Pract 7: 157-160, 2016.

7. Shafgat S, Memon SB, Hyder S, Hasan SH, Smego RA Jr. Brainstem encephalitis with Kikuchi-Fujimoto disease. J Coll Physicians Surg Park 13: 663-664, 2003.

8. Guéguen A, Sené T, Maillart E, Gout O. Encephalitis and CSF increased level of interferon- $\alpha$ in Kikuchi-Fujimoto disease. BMJ Case Rep 27: 2012, 2012.

9. Byun JH, Park SE, Nam SO, et al. Three children of meningoencephalitis with Kikuchi necrotizing lymphadenitis. Brain Dev 40: 251-255, 2018

The Internal Medicine is an Open Access journal distributed under the Creative Commons Attribution-NonCommercial-NoDerivatives 4.0 International License. To view the details of this license, please visit (https://creativecommons.org/licenses/ by-nc-nd/4.0/).

(C) The Japanese Society of Internal Medicine Intern Med Advance Publication 